\title{
Local axonal conduction delays underlie precise timing of a neural sequence
}

Robert Egger $^{1,2} ¥$, Yevhen Tupikov ${ }^{3}$, Kalman A. Katlowitz ${ }^{1,2}$, Sam E. Benezra ${ }^{1,2}$, Michel A. Picardo ${ }^{1,2}$, Felix Moll ${ }^{1,2}$, Jörgen Kornfeld ${ }^{4}$, Dezhe Z. Jin ${ }^{3}$, Michael A. Long ${ }^{1,2} \dagger^{*}$

1) NYU Neuroscience Institute and Department of Otolaryngology, New York University Langone Medical Center, New York, NY 10016 USA

2) Center for Neural Science, New York University, New York, NY, 10003 USA

3) Department of Physics and Center for Neural Engineering, Pennsylvania State University, University Park, Pennsylvania 16802 USA

4) Max Planck Institute of Neurobiology, 82152, Martinsried, Germany

7 Main Figures

2 Supplementary Figures

* To whom correspondence should be addressed: Michael A. Long (mlong@med.nyu.edu)

$\dagger$ Lead Contact

$¥$ Authors contributed equally 


\section{SUMMARY}

43 Sequential activation of neurons has been observed during various behavioral and cognitive

44 processes and is thought to play a critical role in their generation. Here, we studied a circuit in

45 the songbird forebrain that drives the performance of adult courtship song. In this region, known

46 as HVC, neurons are sequentially active with millisecond precision in relation to behavior. Using

47 large-scale network models, we found that HVC sequences could only be accurately produced if

48 sequentially active neurons were linked with long and heterogeneous axonal conduction delays.

49 Although such latencies are often thought to be negligible in local microcircuits, we empirically

50 determined that HVC interconnections were surprisingly slow, generating delays up to $22 \mathrm{~ms}$.

51 An analysis of anatomical reconstructions suggests that similar processes may also occur in rat

52 neocortex, supporting the notion that axonal conduction delays can sculpt the dynamical 53 repertoire of a range of local circuits.

54

55 KEYWORDS: network, conduction delay, sequence, model, motor control, local circuits 


\section{INTRODUCTION}

58 Sequential neural activity in local brain areas is thought to play a critical role in behaviors such as motor control (Luczak et al., 2015; Mauk and Buonomano, 2004; Peters et al., 2014; Prut et al., 1998), navigation (Foster and Wilson, 2007; Pastalkova et al., 2008), and decision-making underlie the generation of neural sequences (Diesmann et al., 1999; Fiete et al., 2010; Goldman, 2009; Hahnloser et al., 2002; Kleinfeld and Sompolinsky, 1988; Laje and Buonomano, 2013; Rajan et al., 2016), but experimental tests of these network models have been stymied by the scarcity of data sets that relate behavior, network function and circuit structure. The zebra finch is an advantageous model organism for studying the network basis of neural sequence generation. Each adult male zebra finch produces a courtship song that is nearly identical from one rendition to the next, consisting of $~ 3-7$ discrete vocal elements known as 'syllables' (Figure 1A). Many lines of evidence have suggested that neural activity controlling the moment-tomoment timing of song production is localized to a single brain region, called HVC (proper name) and is driven by premotor neurons in that region (Figure 1B) (Hahnloser et al., 2002;

72 Long and Fee, 2008; Nottebohm et al., 1976; Vu et al., 1994). HVC premotor neurons produce

73 high-frequency bursts of action potentials ( 4-5 spikes/burst, $\sim 10 \mathrm{~ms}$ duration) at a single 74 moment during the song (Hahnloser et al., 2002) with millisecond precision across song 75 renditions (Figure 1C). At the network level bursts form a sustained sequence spanning the 76 duration of song syllables (Figure 1D and 1E). 
studies had demonstrated direct excitatory connections between premotor neurons (Figure 1F),

81 but the sufficiency of these connections for propagating activity from earlier to later steps in a

82 sequence remains controversial (Cannon et al., 2015; Galvis et al., 2018; Gibb et al., 2009;

83 Hamaguchi et al., 2016; Jin et al., 2007; Long et al., 2010; Pehlevan et al., 2018). For instance,

84 activity may be driven through single strong connections (Lorteije et al., 2009) or through

85 convergent inputs from several presynaptic partners (Bruno and Sakmann, 2006). Two primary

86 lines of evidence support the latter possibility. Local excitatory synaptic strength, as estimated by

87 active zone size in our previous ultrastructural work (Kornfeld et al., 2017), is not extraordinarily

88 large, which does not support the idea of single presynaptic partners. Consistent with this view,

89 unitary synaptic potentials measured with paired intracellular recordings $(\sim 2 \mathrm{mV})$ are

90 considerably smaller than the depolarization observed during singing $(\sim 10 \mathrm{mV})$, suggesting that

91 many presynaptic elements are involved in the generation of spiking events (Kosche et al., 2015;

92 Long et al., 2010; Mooney and Prather, 2005). Although these presynaptic partners are likely to

93 be other premotor neurons within HVC, their exact identity (i.e., spatial location, specific timing)

94 remains unknown. Therefore, in order to take a step towards resolving the present conflicting

95 theories about sequence generation in $\mathrm{HVC}$, it is necessary to understand the nature of this

96 functional convergence.

97

98 To address this issue, we used a network model constrained by experimental measurements of

99 HVC premotor neuron properties and population activity. We found that the spatiotemporal

100 organization of the HVC sequence during singing is best matched by a neural network

101 architecture in which neurons are connected by local axon collaterals with long conduction

102 delays - matching those observed in HVC. Because of these heterogeneous conduction delays, 
103 sequential activity propagates via convergent input from presynaptic neurons active at different

104 times and, as a result, activity is 'polychronous' (Izhikevich, 2006) - generating continuous time-

105 locked spiking patterns without synchrony. To assess the significance of local axonal delays in

106 other brain areas, we estimated conduction delays along axons of layer 4 (L4) neocortical

107 neurons (Narayanan et al., 2015), and find that the differences in axonal arbor size and

108 conduction velocity compensate and yield delays similar to those observed in zebra finch HVC.

109 Hence, axonal conduction delays may play a key role in shaping network activity within local

110 brain circuits. 


\section{RESULTS}

\section{Synfire chain models do not explain HVC population data}

114 An effective mechanism for generating postsynaptic convergence is the synchronous activation

115 of presynaptic neurons (Figure 2A) (Bruno, 2011; Bruno and Sakmann, 2006). Feedforward 116 networks based on synchronous neuronal activation, known as 'synfire chains', have long been 117 suggested to underlie sequence generation (Abeles, 1991; Amari, 1972). This network 118 architecture has previously been proposed to explain HVC sequences (Fee et al., 2004; Fiete et 119 al., 2010; Jin et al., 2007; Long et al., 2010; Pehlevan et al., 2018), and has the benefit of 120 capturing the precision of individual neurons and the stability of network sequences (Jin et al., 121 2007; Long et al., 2010). We generated a synfire chain network model of 20,000 active HVC 122 premotor neurons (Long et al., 2010), where HVC premotor neurons were triggered by 123 synchronously active presynaptic ensembles (see Methods), as reflected in the fine structure of 124 the network output (Figure 2B, i.e., discrete groups of synchronously active neurons with $\sim 5-6$ $125 \mathrm{~ms}$ intervals between burst onset times). As before, this model produced a sustained network 126 sequence covering syllable-length timescales.

128 Until recently, such network models were difficult to test empirically; the number of recordings 129 performed in HVC during song production were limited (Amador et al., 2013; Hahnloser et al., 130 2002). However, following recent improvements in recording technology, we can now track the 131 activity of large cell populations (Lynch et al., 2016; Okubo et al., 2015; Picardo et al., 2016) to 132 test model predictions against the data. Therefore, we looked for this pattern of synchronized 133 burst onset times in HVC by examining a recently reported data set of 286 projection neurons in 1345 birds measured using extracellular recordings during singing (Figure 2C and 2D) (Lynch et al., 
2016). We compared these measurements with predictions from the synfire chain model (Figure $2 \mathrm{E}$ and $2 \mathrm{~F}$ ) and found that the timing of activity was qualitatively different: the synfire model predicted that activity would be clustered at distinct timepoints, while the recorded data appeared to lack such temporal structure. To obtain a quantitative comparison of these differences, we computed the power spectrum of burst onset times. The synfire chain model predicted a peak in the power spectrum at $\sim 180 \mathrm{~Hz}$, corresponding to the $\sim 5-6 \mathrm{~ms}$ interval between synchronous groups of neurons (Figure 2B), while the recorded data exhibited a flat power spectrum, consistent with a more uniform distribution of burst times (Figure 2G). Therefore, HVC activity does not appear to be restricted to synchronous groups, suggesting that the network connectivity is not organized as a synfire chain.

\section{Temporal structure of polychronous network sequences is affected by conduction delays}

In an alternative model, presynaptic neurons are active across a range of different times, and heterogeneous network delays are sufficient to allow spikes from multiple neurons to arrive simultaneously at a postsynaptic target (Figure 3A) (Izhikevich, 2006). Previous theoretical work based on conduction delays measured between different brain areas (Swadlow, 1985, 1994) demonstrated that such a 'polychronous' network architecture can generate time-locked spiking patterns without synchrony (Bienenstock, 1996; Izhikevich, 2006), but it is unclear if delays in local circuits are sufficient to generate sustained sequences in this context. To estimate these delays, we needed a measure for axonal pathlength as well as conduction velocity. Axonal pathlengths to each synapse were determined using previous observations from our laboratory; the relative position and abundance of synapses were measured using electron microscopy (Kornfeld et al., 2017) and the total axonal extent for each neuron was obtained from 22 
complete light microscopic reconstructions of local axonal collaterals (Figure 3B) (Benezra et al., 2018). Our first estimate for conduction velocity was $0.3 \mathrm{~mm} / \mathrm{ms}$, a value reported for local unmyelinated axons in mammalian neocortex (Helmstaedter et al., 2008; Hirsch and Gilbert, 1991; Shu et al., 2007). Using these parameters, we developed a procedure to generate a feedforward polychronous network of HVC premotor neurons for a given distribution of axonal conduction delays (Figure S1). Because measured pathlength distances follow a log-normal distribution (Buzsaki and Mizuseki, 2014), we use this shape as our first estimate of these delays (Figure S1F). We find that the resulting network produces a reliable sequence in which neurons are active in a continuous fashion with burst onset times spread throughout (Figure 3C), thus matching our empirical observations (Figures 2E, 3D and 3E), in contrast to the discrete synchronous activity of the synfire chain model (e.g., Figure $2 \mathrm{~F}$ and $2 \mathrm{G}$ ).

How important are axonal conduction delays in the function of the polychronous network? To examine this question, we artificially increased the conduction velocity of local axons by an order of magnitude within our network model. When we made the axonal delays ten times shorter (Figure 3F), we found that the resulting network sequence consisted of groups of neurons bursting near-synchronously (Figure 3F-3H), as in the synfire chain model. This result suggests that the nature of sequential activity can qualitatively shift based on the distribution of axonal conduction delays. To explore this issue more thoroughly, we created a range of models in which the means and standard deviations (SD) of axonal delays were varied parametrically over an order of magnitude (Figures 4A, 4B, and S1F), encompassing the examples discussed above (Figure 3). For each combination of mean and SD, we generated a polychronous network model and simulated network activity. We then compared our model with experimental observations by 
181 determining whether the degree of network synchrony in each model - as determined by the

182 power spectrum of burst onset times (Figure 4C and 4D) - was significantly different than

183 empirical measurements (Figure 4E and 4F). The resulting map of the parameter space of the

184 polychronous network revealed two regions which could be distinguished by the experimentally

185 observed distribution of burst onset times (Figure 4G). Where the delay distribution is narrow

186 and/or the mean delay very small, sequences are based on synchronized groups of neurons,

187 similar to a synfire chain, and thus incompatible with HVC dynamics. Above a minimum mean

188 and SD of the delay distribution, polychronous network sequences are continuous, matching

189 song-related activity (e.g., Figure 2E). These activity patterns are a consequence of the

190 requirement of convergence at the postsynaptic neuron and the range of conduction delays from

191 presynaptic neurons. In cases where there are heterogeneous delays (e.g., Figure 3C), inputs can

192 converge onto the same postsynaptic neuron from presynaptic neurons active at different times,

193 enabling continuous sequences. In contrast, if the range of delays is very narrow (e.g., Figure

$1943 \mathrm{~F})$, convergent inputs necessarily originate from presynaptic neurons active within this very

195 narrow range, resulting in a sequence consisting of synchronous groups of neurons.

196

197 Slow conduction velocity of local HVC axon collaterals

198 Our polychronous network model puts a strong lower bound on the conduction delays that must 199 exist in the HVC circuit, and our value for axonal delays, based upon published mammalian 200 conduction velocities, is close to the boundary between continuous and discrete network 201 sequences (Figure 4G), resulting in mild, transient synchronous activity in the beginning of the 202 sequence (Figure 3C). We therefore decided to obtain a more precise estimate of these delays 203 through experimental observation. A direct measure of conduction delays is complicated by the 
204

205

206

207

208

209

210

211

212

213

214

215

216

217

218

219

220

221

222

223

224

225

226

fact that local unmyelinated axons are typically thin and difficult to record (Shu et al., 2006). However, HVC neurons often exhibit long-range unmyelinated axons that target the downstream song production structure (i.e., the robust nucleus of the arcopallium, RA) (Figure 1B and 5A and S2). We reasoned that we could measure the conduction velocities of these fibers and then relate long-range delays to those of the local axons within HVC. We measured the conduction velocity of action potentials in the $\mathrm{HVC} \rightarrow \mathrm{RA}$ projection axons by quantifying the time required for an antidromic spike initiated in RA to travel to the soma (Figure 5A, S2A and S2B) (Hahnloser et al., 2006), a path distance of $2.8 \pm 0.2 \mathrm{~mm}(\mathrm{n}=4$ reconstructions, Figure 5B). We then compared the morphological properties of descending axons - restricting our view to only the unmyelinated fibers (Figure S2C and S2D) - with another EM data set in which local axons of HVC premotor neurons were labeled (Figure 5C and 5D). Local axons were invariably unmyelinated and significantly thinner $(167 \pm 73 \mathrm{~nm})$ than unmyelinated descending axons (446 $\pm 135 \mathrm{~nm}$ ) (Figure 5D, S2E and S2F). Assuming that biophysical properties of the unmyelinated local and long-range axons are similar, we used cable properties to convert the conduction velocity measurements of the descending axons to those of local HVC collaterals (Hodgkin and Huxley, 1952; Rushton, 1951). The estimated conduction velocity of HVC axon collaterals $(0.187 \pm 0.035 \mathrm{~mm} / \mathrm{ms})$ enabled us to infer the propagation time for spikes to travel from the soma to different parts of the axon (Figure 5E). Using this estimate, we found delays ranging from 1 to $7.5 \mathrm{~ms}\left(5^{\text {th }}\right.$ and $95^{\text {th }}$ percentiles) and up to $22 \mathrm{~ms}$. This more precise estimate of the conduction velocity is considerably slower than our previous assumption and places HVC comfortably within the parameter region of polychronous networks producing continuous sequences (Figures 5F and S3). 


\section{Polychronous network organization explains HVC spatiotemporal activity}

230 To this point, we have demonstrated that the polychronous model given our measured experimental constraints can explain the temporal structure of HVC function at a network (i.e., continuous representation) as well as at a cellular (i.e., axonal conduction delays) level. We next asked whether this underlying circuit structure can predict other aspects of song-related HVC function. To accomplish this, we returned to our local axonal collateral reconstructions, and we placed synapses at specific locations within the axonal field that fit a variety of conduction delay exhibited a low variance, synapses were clustered on distal axons (Figure 6A). We can also look at cases in which the means of the conduction delays were low, across two variance conditions

239 (Figures 6B and 6C). We compare these possibilities against a scenario that matches our experimental observations in which the mean and variance were both relatively high (Figure 6D). Because of the differential placement of synapses within the axonal field, each model should result in a different prediction concerning the spatiotemporal pattern of activity in HVC during

243 singing (Graber et al., 2013; Markowitz et al., 2015; Peh et al., 2015). We performed 2-photon

244 imaging of GCaMP6-expressing projection neurons during singing to measure the activity of 182

245 putative premotor neurons (see Methods), combining new observations with a previously 246 published data set (Katlowitz et al., 2018; Picardo et al., 2016) (Figure 6E). Using an established 247 algorithm, we precisely estimated burst onset times with a temporal resolution of $\sim 10 \mathrm{~ms}$ 248 (Picardo et al., 2016) and related these values to the relative spatial position for each neuron 249 (Figure 6F). We excluded pairs in which the difference in burst times was greater than $20 \mathrm{~ms}$ and 
therefore unlikely to be driven by monosynaptic connections. In the remaining cases, sequentially active neuron pairs were found over a wide range of relative locations $(178 \pm 102$ $\mu \mathrm{m}$, mean $\pm \mathrm{SD})$, from immediately adjacent $(\sim 10 \mu \mathrm{m})$ to much longer distances $(\sim 500 \mu \mathrm{m}$, or approximately one third of the maximum extent of HVC) (Figure 6G and 6H). We then compared the spatial location of sequentially active pairs against the predictions of our

previously stated models. Whereas three models predicted a high degree of spatial clustering

(Figure 6A-6C), the model based on our empirically measured delay distributions was more

spatially dispersed (Figure 6D), matching the functional data (Figure 6I). We conclude that a

polychronous network sequence based on conduction delays observed in HVC results in a spatial

organization that closely resembles observations of HVC network activity during song.

\section{Delay distributions are conserved from songbird to mammalian neocortex}

262 We have demonstrated an important impact of local axonal delays on the timing and structure of network activity within HVC of the zebra finch. Given the extraordinarily slow axonal conduction velocity in HVC compared with known values measured in a variety of different

265 circuits (Figure 7A), it remains unclear whether such delays will play a role within those networks or whether this solution is simply a specialization within zebra finch HVC. To begin to

267 examine this issue, we analyzed the local collaterals of 14 spiny neurons in Layer 4 of rat 268 somatosensory cortex (Figure 7B) (Narayanan et al., 2015). When we measured the pathlength 269 from the soma to different locations along the axon, we found that the entire size of the axonal 270 field was considerably larger than that of HVC premotor neurons (Figure 7C). Surprisingly,

271 when we estimated conduction delays - accounting for both the discrepancies in conduction 272 velocity and pathlength - we find that the range of these values is identical in both cell classes 
273 (L4: $3.4 \pm 2.3 \mathrm{~ms}$, mean \pm SD; HVC: $3.3 \pm 2.1 \mathrm{~ms}$, Figure 7D). Therefore, significant conduction

274 delays exist within the rodent neocortex, potentially playing an important computational role

275 within that circuit. 


\section{DISCUSSION}

277 Using a range of modeling and experimental approaches, we investigated how local excitatory

278 circuits can give rise to convergent synaptic input underlying sequential activity in the zebra

279 finch song system. We provided three independent lines of evidence supporting a central role for

280 slow and heterogeneous axonal conduction delays in HVC sequence generation. First, network

281 modeling revealed that delays are required to generate the continuously active population

282 sequences observed in HVC. Second, the delays predicted by the network model match

283 empirically measured values for local HVC axon collaterals. Third, the spatiotemporal patterns

284 of HVC activity observed during singing matches the predictions from our model. As a result,

285 we propose that the core circuit for sequence generation in HVC consists of an asynchronous

286 feedforward network based on a variety of conduction delays to generate a continuous neural

287 sequence. Notably, previous theoretical work has demonstrated that axonal delays in synfire

288 chain networks can enable more continuously active network sequences by 'skipping'

289 connections between different groups, therefore enabling so-called 'synfire braid' networks

290 (Bienenstock, 1996) which function similarly to polychronous networks. Future studies will

291 elucidate how these sequences are started (Figure S3) (Andalman et al., 2011; Danish et al.,

292 2017; Galvis et al., 2018) as well as the role of other circuit elements, such as local circuit

293 interneurons, in this process (Gibb et al., 2009; Jin et al., 2007; Kosche et al., 2015; Yildiz and

294 Kiebel, 2011).

296 In this study, we provide the first demonstration of a biological neural network implementing the

297 polychronous circuit structure. We find that this network is capable of producing sustained

298 sequential activity over behaviorally relevant timescales (Itskov et al., 2011), exceeding the 
predictions from the original model. The continuous neural sequences arising from this model can represent any moment in time, thereby greatly increasing the resolution of the HVC premotor clock and facilitating the placement of descending motor commands at any time point during the song. While the present model assumes that synaptic connections are made within a synchronous time window, another feature of polychronization is the potential to self-organize such connectivity patterns through spike timing-dependent plasticity mechanisms (Gerstner et al., 1996; Izhikevich, 2006), and future work will determine the relevance of axonal delays for assembly of HVC circuits during song learning (Fiete et al., 2010; Jun and Jin, 2007; Okubo et al., 2015).

We found that slow axons within a local circuit are critical for continuous sequence generation. We estimate that conduction delays are significantly larger than those afforded by other biophysical parameters, such as synaptic delays (Sabatini and Regehr, 1996) ( 0.1 ms) and postsynaptic integration time (Long et al., 2010) ( 5 ms during singing). Notably, the dendrites of HVC premotor neurons are highly compact (i.e., a spatial extent of less than $200 \mu \mathrm{m})($ Benezra et al., 2018) and are unlikely to contribute significantly, although to date no direct measurements of their electric signaling properties exist (Magee and Cook, 2000; Williams and Stuart, 2002). Therefore, approximately half of the total elapsed time of the HVC sequence could be attributed to local axonal conduction, a comparatively inflexible process that may underlie the behavioral stereotypy inherent in the adult zebra finch song (Lombardino and Nottebohm, 2000) by rendering the circuit less sensitive to perturbations (Hamaguchi et al., 2016; Swadlow et al., 1981). We anticipate that further studies will clarify the impact of additional time delays introduced along the pathway from HVC to syringeal motorneurons (Figure S2A) in converting 
322 the HVC code to behavior and the extent to which the axonal properties themselves (e.g.,

323 myelination status or axial diameter) can be modified through experience. Furthermore, although

324 our model does take into account a range of axonal diameters for local collaterals, the detailed

325 effects of precise axonal morphology - e.g., possible failures at branch points (Swadlow et al.,

326 1980) - remain to be explored.

327

328 The idea that axons contribute to information processing in neural circuits has long been

329 explored for long-range connections between different brain areas (Carr and Konishi, 1988;

330 Innocenti et al., 1994; Salami et al., 2003; Sugihara et al., 1993). For instance, in the brainstem

331 of the barn owl, axons carrying sound information from both ears form precisely tuned and

332 spatially organized 'delay lines' (Jeffress, 1948) necessary for detecting minute interaural time

333 differences (Carr and Konishi, 1988). In contrast, the role of axonal delays within local

334 microcircuits is often disregarded (Budd et al., 2010), possibly because of the technical

335 challenges involved in obtaining reliable estimates of conduction velocity in local circuits. In this

336 study, we find that the premotor song production structure HVC in the zebra finch uses 'delay

337 lines' within a local circuit to generate reliable sequences of activity during song (Katlowitz et

338 al., 2018). We do not yet know whether this specialization is unique to circuits in which a high

339 degree of temporal precision is required or more broadly found in other networks, including

340 those capable of more flexibility. Although we find that conduction delays along intracortical

341 axons in a rodent neocortical area are likely to be comparable to those reported here in zebra

342 finch $\mathrm{HVC}$, the extent to which these collaterals may support persistent activity that has been

343 observed within this region so far remains unexplored (Sachidhanandam et al., 2013). Further

344 work in other circuits can establish whether this delay distribution represents a universal scaling 
345 law (Buzsaki and Mizuseki, 2014; Liewald et al., 2014; Miller, 1996) across different species,

346 brain regions, cell types, etc., or whether these local delays are specially tuned for the

347 requirements of each unique case. Overall, our results suggest that in addition to defining the

348 static architecture of neural networks (Denk et al., 2012; Plaza et al., 2014; Seung, 2012),

349 functional properties of axons within local circuits can also control the space of neural activity 350 patterns.

351 


\section{ACKNOWLEDGEMENTS}

353 We thank Dmitriy Aronov, György Buzsáki, Dmitri Chklovskii, Yarden Cohen, Annegret

354 Falkner, Dan Levenstein, Cengiz Pehlevan, Alex Reyes, John Rinzel, Richard Tsien, and 355 members of the Long laboratory for comments on earlier versions of this manuscript. We thank 356 the Fee laboratory for providing extracellular recordings of HVC projection neurons and Marcel

357 Oberlaender for the reconstructions of L4 neurons. We thank NYU Langone's Microscopy 358 Laboratory for assistance with electron microscopy. We also acknowledge helpful conversations 359 with Asohan Amarasingham, Yoram Burak, Dina Obeid, and Kanaka Rajan. Funding: This 360 research was supported by the DFG EG 401/1-1 (R.E.), NIH R01 NS075044 (M.A.L.), NSF EF3611822478 (D.Z.J. and M.A.L.), Simons Global Brain (M.A.L.).

\section{AUTHOR CONTRIBUTIONS}

364 R.E. and M.A.L conceived the study and designed the experiments; S.E.B., M.A.L., M.A.P, F.M. 365 and J.K. conducted the research; R.E., K.A.K., S.E.B., M.A.P., F.M., J.K., and M.A.L. 366 performed data analyses; Y.T. and D.Z.J. developed the theoretical model; R.E., Y.T., K.A.K., 367 and M.A.L. created the figures; R.E. wrote the initial draft of the manuscript; R.E., D.Z.J., and 368 M.A.L. edited and reviewed the final manuscript. R.E., M.A.L., and D.Z.J. acquired funding;

369 M.A.L. and D.Z.J. supervised the project.

\section{DECLARATION OF INTERESTS}

372 The authors declare no competing interests. 
HVC and its downstream target along the song production pathway, the robust nucleus of the arcopallium (RA). RA sends projections to brainstem motoneurons involved in producing vocalizations. (C) Spectrogram of an individual syllable (top) and spike raster plots of an HVC premotor neuron during different song renditions (middle) (Lynch et al., 2016). Bottom: relative neurons aligned relative to on- offset of the syllable during which they are active. Red: example spike train from (C). (E) Distribution of syllable durations (58 syllables from 14 birds). (F) neurons. Black circle: soma location; grey spheres: modeled location of synapses onto other HVC premotor neurons along the axon. synaptic connections onto the same postsynaptic neuron, resulting in a sustained sequence at the population level. (B) Spike raster plot of sequential activity in a synfire chain model of HVC 394 premotor neurons with numbers of synaptic connections constrained by anatomical 395 measurements. Spikes from $10 \%$ of all active neurons are shown. Inset: Magnified view of spike 396 raster plot highlighted $(*)$, revealing synchronously active groups of neurons. (C) Top: 

neuron activity recorded during song. Grey: syllables. (D) Burst onset times in (C) relative to onand offset of the syllables during which they occur. (E) Burst onset times of HVC projection neurons relative to syllable on- and offset in 23 syllables from 5 birds (Lynch et al., 2016). matching duration and number of observed burst times for each syllable. (G) Power spectrum of burst onset times calculated from experimental observations and the synfire chain model. Shaded area: \pm 3 SD (bootstrap).

polychronous network models. (A) The activity of neurons forming convergent synaptic connections onto the same postsynaptic neuron does not have to be synchronized. Instead, if differences in spike times are compensated by suitable delays, these spikes arrive synchronously at the postsynaptic neuron, resulting in a network sequence without synchrony. (B) Pathlength to soma measured along the reconstruction of local axon collaterals of an HVC premotor neuron.

412 Inset: Pathlength distribution measured for 22 HVC premotor neuron axons. (C) A polychronous

413 network model of HVC premotor neurons connected by synapses with conduction delays (top 414 inset) based on axonal pathlengths and a conduction velocity of $0.3 \mathrm{~mm} / \mathrm{ms}$ generates a sustained 415 sequence of bursting activity. Bottom inset: Burst onset times are distributed continuously 416 throughout the sequence. Spikes from $10 \%$ of all active neurons are shown. (D) Burst onset 417 times predicted by the polychronous network model by matching duration and number of 418 observed burst times for each syllable. (E) Power spectrum of burst onset times calculated from 419 the observed burst onset times and the polychronous network model. Shaded area: \pm 3 SD 
420 (bootstrap). (F) A polychronous network model of HVC with ten times smaller conduction

421 delays compared to the delays in $\mathrm{C}$ (top inset) results in a sequence of bursting activity of HVC

422 premotor neurons in which bursts occur in synchronous groups of neurons (bottom inset). (G, H)

423 As in D and $\mathrm{E}$ for the above model in $\mathrm{F}$.

424

425

426

Figure 4. Polychronous network models support two distinct network sequence patterns depending on the parameters of the underlying delay distribution. (A) Three distributions of

427 conduction delays with different means and identical variances (SD: $1.25 \mathrm{~ms}$ ). (B) Three 428 distributions of conduction delays with identical means (mean: $3.5 \mathrm{~ms}$ ) and different variances.

429 (C, D) Generating a polychronous network model for each distribution (C: varying the mean; D: 430 varying the SD) allows investigating which conduction delay distributions result in continuous 431 network sequences or sequences with synchronous groups of neurons. (E, F) Mean power in the 432 frequency band from $75-200 \mathrm{~Hz}$ for the three models in (C) and (D) and the observed burst times 433 (black line) allows measuring the degree of synchrony in the different networks. Error bars: $5^{\text {th }}$ 434 and $95^{\text {th }}$ percentiles (bootstrap). (G) Two-dimensional parameter grid of polychronous networks 435 with different mean and SD of delay distributions investigated here. Each grid point is colored 436 according to the mean power of the burst onset times to determine whether the underlying 437 network produces continuous network sequences (i.e., low power, indicated in dark blue) or 438 sequences with synchronous groups of neurons (i.e., high power, indicated in green and yellow). 439 White/black: Location of the models in Figure 3B (1) and 3F (2) on the parameter grid. Black 440 line: Models to the left and below this line display sequences with synchronously active groups 441 of neurons that are not observed in $\operatorname{HVC}(\mathrm{p}<0.05$, bootstrap). 
443 Figure 5. Local HVC premotor neuron axons have conduction delays supporting continuous sequences. (A) Antidromic stimulation of HVC premotor neuron axons and wholecell recording at the soma allows precise measurement of conduction times along the descending axon. Stimulus artifact is blanked for visualization. (B) Example reconstructions of the projection axon of a premotor neuron connecting HVC and RA. (C) 3D reconstruction of the soma and proximal axons of a retrogradely labeled HVC premotor neuron from an SBEM image stack. Insets: EM micrographs of labeled axons. (D) Unmyelinated axons in the HVC-RA fiber tract have larger diameters than local (unmyelinated) collaterals of HVC premotor neurons. (E) Measurement of the conduction velocity along unmyelinated long-range axons allows a precise estimate of the conduction delays along thin unmyelinated local axon collaterals of HVC premotor neurons based on 22 reconstructions of local axon collaterals. (F) The distribution of conduction delays along HVC premotor neuron axons supports a polychronous network model generating continuous sequences (see Figure 4G).

462 photon calcium imaging of song-related bursting activity in HVC. Left: Example image of 463 GCaMP6s-labeled somata. Right: Spectrogram of song motif (top), aligned normalized 464 fluorescence traces of the neuron highlighted in left panel (center), and estimated burst onset 465 time (bottom). (F) Left: Soma locations of 18 neurons active within the same syllable, projected 
466

467

468

469

470

471

472

473

474

475

476

477

478 in (A). D/V: dorsal/ventral. (C, D) Distributions of axonal pathlengths (C) and resulting 479 conduction delays (D) for $22 \mathrm{HVC}$ premotor neuron axons and 14 L4 spiny neuron axons 480 (Narayanan et al., 2015).

onto the horizontal plane. Right: Estimated burst onset times of the same neurons within the syllable. (G) Relative soma locations of putatively connected neurons in F (i.e., burst onset times within $20 \mathrm{~ms}$ of each other). (H) Relative soma locations of putatively connected neurons in nine birds. (I) Radial distribution of putative postsynaptic neurons in (H) (dashed line) and radial distribution of active synapses predicted by the four network models in A-D (solid lines).

Figure 7. Conduction delays of neocortical axonal arbors. (A) Axonal conduction velocity measurements from the peripheral to the central nervous system. Peripheral nerves: (Hursh, 1939); squid giant axon: (Hodgkin and Huxley, 1952); nucleus magnocellularis axons: (Carr and Konishi, 1990); intracortical axons: (Hirsch and Gilbert, 1991; Shu et al., 2007). (B) Example reconstructions of an HVC premotor neuron axon and a L4 spiny neuron axon from rat somatosensory cortex. Conduction delays estimated based on conduction velocity measurements 
STAR METHODS

483

484

CONTACT FOR REAGENT AND RESOURCE SHARING

485 Further information and requests for resources and reagents should be directed to and will be 486 fulfilled by the Lead Contact, Michael Long (mlong@ med.nyu.edu).

487

488

\section{EXPERIMENTAL MODEL AND SUBJECT DETAILS}

We used adult (>90 days post hatch) male zebra finches (Taeniopygia guttata) that were obtained from an outside breeder and maintained in a temperature- and humidity-controlled environment with a 12/12 hr light/dark schedule. All animal maintenance and experimental procedures were

492 performed according to the guidelines established by the Institutional Animal Care and Use 493 Committee at the New York University Langone Medical Center.

\section{METHOD DETAILS}

\section{Surgeries}

497 Surgical procedures for retrograde labeling of HVC premotor neurons, viral injections, chronic 498 cranial window implantation for 2-photon imaging, and in vivo whole cell recordings, have 499 previously been described in detail (Kornfeld et al., 2017; Long et al., 2010; Picardo et al., 500 2016). Briefly, animals were anesthetized (1-3\% isoflurane in oxygen) and the scalp was cut to 501 expose the entire skull. To label HVC premotor neurons for electron-microscopic imaging, a 502 biotinylated dextran (BDA-dextran, MW: 3,000; Invitrogen) was injected into RA. Birds were 503 allowed to recover for three days to allow retrograde labeling. For virus injections, a craniotomy 504 was made over HVC and either AAV9.Syn.GCaMP6s.WPRE.SV40 (Penn Vector Core) or a 1:1 

amplitude, bipolar stimulation of $0.2 \mathrm{~ms}$ duration).

523

\section{$524 \quad$ 2-photon calcium imaging}

525 The procedures for 2-photon calcium imaging of HVC neurons during singing have been

526 described previously (Katlowitz et al., 2018; Picardo et al., 2016). Briefly, birds were first

527 trained to perform directed singing in the head-fixed configuration upon presentation of a female 
bird using operant conditioning with a water reward (Picardo et al., 2016). Once the behavior was learned, virus injections and cranial window implantation were performed. 2-photon calcium imaging was carried out using a resonant scanning system (Thorlabs) at a frame rate of $28.8 \mathrm{~Hz}$ and a 16x water-immersion objective (NA 0.8, WD $3 \mathrm{~mm}$; Nikon). We acquired singing behavior using an omnidirectional microphone (Audio-Technica) digitized at $40 \mathrm{kHz}$ (Digidata 1550, Molecular Devices). Motif-related image data were temporally aligned offline by linearly warping to manually annotated reference points within the song. Fluorescence traces were extracted from manually drawn ROIs (ImageJ) on temporally aligned and motion-corrected (Miri et al., 2011) image stacks. Frame times were defined for each neuron as the mean time point that the laser reached the ROI as a function of vertical scanning location. Last, burst onset times were deconvolved from the raw traces using a Markov Chain Monte Carlo inference approach with an average uncertainty of $\sim 10 \mathrm{~ms}$ (Picardo et al., 2016; Pnevmatikakis et al., 2016). These analyses were performed using custom Matlab scripts (Mathworks).

\section{Extracellular recordings during singing}

We reanalyzed a previously reported data set of burst times from HVC neurons during singing (Lynch et al., 2016; Okubo et al., 2015). The data set contained extracellular recordings obtained in two adult birds (i.e., $\geq 103$ d.p.h.) and three young adult birds (i.e., $\geq 59$ d.p.h.) with a stable motif. Single units were identified as HVC projection neurons (i.e., projecting to nucleus RA or along the anterior forebrain pathway to Area X) by antidromic stimulation or as putative HVC projection neurons based on low spontaneous firing rate (i.e., $<1 \mathrm{~Hz}$ ) and sparse bursting activity during singing. Individual song motifs and the accompanying neural activity were time-warped to syllable on- and offsets. Then, the firing rate was computed in $1 \mathrm{~ms}$ bins and smoothed with a 
5519 ms wide sliding window. A 'burst window' was defined as a period where the smoothed firing

552 rate exceeded a threshold of $10 \mathrm{~Hz}$. To define the burst onset more precisely, each spike falling

553 into the 'burst window' was replaced with a $5 \mathrm{~ms}$ wide 'spike interval' starting at the spike time.

554 The burst onset time is defined as the earliest time point in a 'burst window' where at least three

555 'spike intervals' from different song renditions overlap.

556

557 Histological procedures

558 For serial block-face electron-microscopic (SBEM) imaging, perfusion and histology was

559 performed as described in detail previously (Kornfeld et al., 2017). For transmission electron-

560 microscopic imaging, the protocol used for SBEM imaging was slightly modified as follows.

561 After the bird was transcardially perfused, the brain was removed from the skull and post-fixed

562 overnight (Kornfeld et al., 2017). The brain was then cut into $100 \mu \mathrm{m}$ thick slices using a

563 vibratome (Leica VT1000S). Residual peroxidase activity was suppressed by soaking the sample

564 in $3 \% \mathrm{H}_{2} \mathrm{O}_{2}$ for 20 min before labeling the sample with an avidin-peroxidase complex and DAB.

565 A slice containing clearly visible stained fibers from HVC to RA was carefully unmounted by

566 immersing the microscope slide into PB. After washing with PB, the samples were post fixed in

$5671 \% \mathrm{OsO}_{4}$ for 2 hours, block stained with $1 \%$ uranyl acetate for 1 hour, dehydrated in ethanol and

568 embedded in EMbed 812 (Electron Microscopy Sciences, Hatfield, PA). Semi-thin sections were

569 cut at $1 \mu \mathrm{m}$ and stained with $1 \%$ toluidine blue to find the previously identified area of interest

570 containing fibers from HVC to RA. In each sample, 20 serial ultrathin sections with $100 \mathrm{~nm}$

571 thickness were cut, mounted on slot copper grids, and stained with uranyl acetate and lead

572 citrate.

573 


\section{Transmission-electron microscope imaging}

575 Stained grids were examined under a Philips CM-12 electron microscope (FEI; Eindhoven, The

576 Netherlands) and photographed with a Gatan (4k x 2.7k) digital camera (Gatan, Inc., Pleasanton,

577 CA). Samples were imaged at a series of increasing magnifications (i.e., ranging from 3,400x to

$57866,000 x$ magnification) to allow identification of fiber tracts and ultimately individual fibers

579 within these tracts. Diameter measurements of unmyelinated projection axons were made on 580 images with a magnification of at least 40,000x.

\section{Axon diameter measurements}

583 All light micrographs used for illustration of local and descending axons were captured using a

584 Zeiss AxioObserver Inverted. We acquired images of descending HVC premotor neuron axons

585 from ultrathin sections using a transmission electron microscope (see above). Unmyelinated

586 descending axons were identified based on dark DAB labeling in EM micrographs. Myelinated

587 axons were identified morphologically by presence of multiple, closely wrapped membrane

588 layers (i.e., myelin sheaths). Diameters were measured along the shortest axis of the

589 circumference of each axon (i.e., if the axon was cylindrical, this corresponds to the diameter of 590 the cylinder irrespective of sectioning angle) (Figure S2F). Diameters of local HVC premotor 591 neuron collaterals were measured using a previously reported data set acquired using serial 592 block-face EM (Kornfeld et al., 2017) with a voxel size of 11 x 11 x $29 \mathrm{~nm}^{3}$ containing HVC 593 premotor neurons labeled by injection of a tracer (BDA-dextran) into RA. Diameters of 594 randomly selected locations along labeled local axon collaterals were measured by determining 595 the image plane that was closest to the orthogonal plane defined by the axon and measuring the 596 axon diameter in that plane. 


\section{Estimating synapse locations along axons}

599 We estimated the possible locations of synapses between HVC premotor neurons along local 600 axons by combining results from previously reported anatomical data sets (Benezra et al., 2018; 601 Kornfeld et al., 2017). We used a database of 22 reconstructions of local axon collaterals of in 602 vivo labeled HVC premotor neurons (Benezra et al., 2018) to determine possible synapse 603 locations irrespective of the postsynaptic target along the local axons of each neuron by sampling 604 points along the reconstructed axons at the average distance between synapses, which has 605 previously been determined using EM measurements along HVC premotor neuron axons 606 (Kornfeld et al., 2017). We then estimated which of these possible synapse locations could target 607 other HVC premotor neurons. To do so, we fitted previous EM measurements of the relative 608 frequency of HVC premotor neurons as the postsynaptic target as a function of distance to the 609 presynaptic soma with a sigmoidal function. For each estimated synapse location along the 610 reconstructed axons, we then computed the synapse-soma distance and placed a synapse at this 611 location with a probability equal to the corresponding relative frequency. Finally, we computed 612 the pathlength distance between each of these possible premotor synaptic locations and the soma 613 (i.e., the shortest path along the axon connecting these two points).

615 Synapse locations along HVC premotor neuron axons for a given delay mean and SD were 616 estimated as follows. Points along the reconstructed axon were grouped according to their 617 pathlength distance to the soma into $50 \mu \mathrm{m}$ bins. If successive points in the reconstruction had an 618 interval of more than $1 \mu \mathrm{m}$, additional points were inserted at $0.5 \mu \mathrm{m}$ intervals using linear 619 interpolation (i.e., leaving the pathlength unchanged). Next, the log-normal delay distribution 
with given mean and SD was converted to a pathlength distribution by multiplication with the

621 axonal conduction velocity of local HVC premotor neuron axons (i.e., $0.187 \mathrm{~mm} / \mathrm{ms}$ ). For each

622 neuron, we generated $\mathrm{N}_{\mathrm{Syn}} * \mathrm{~L}_{\mathrm{Neuron}} / \mathrm{L}_{\mathrm{Avg}}$ samples from this log-normal distribution. Here, $\mathrm{N}_{\text {Syn }}$

$623=170$ is the average number of synapses made by each HVC premotor neuron onto other

624 premotor neurons, $\mathrm{L}_{\mathrm{Neuron}}$ is the total axonal pathlength of this specific premotor neuron and $\mathrm{L}_{\mathrm{Avg}}$

625 is the average axonal pathlength across all 22 premotor neurons (Benezra et al., 2018). Samples

626 beyond the maximum pathlength distance to the soma were repeated. A histogram of these

627 samples with a bin width of $50 \mu \mathrm{m}$ was computed. For each $50 \mu \mathrm{m}$ bin, points along the

628 reconstruction in the corresponding pathlength bin were randomly sampled until the number of

629 elements in this bin of the histogram was reached and a synapse was placed at the location of 630 each sampled points along the reconstruction.

632 Estimating axonal conduction delays

633 The first estimate of conduction delays along local HVC premotor neuron axons was obtained by

634 measuring the pathlength from the soma to points along the axon spaced at the mean inter-

635 bouton interval of HVC premotor neuron axons (10.5 $\mu \mathrm{m})$ (Kornfeld et al., 2017). Combining

636 these measurements from 22 reconstructions of premotor neuron axons reported previously

637 (Benezra et al., 2018) resulted in and average distribution of pathlengths. We then converted 638 these pathlengths into a conduction delay distribution by multiplying each pathlength distance 639 with a value of $0.3 \mathrm{~mm} / \mathrm{ms}$ for the conduction velocity of unmyelinated neocortical axons. A log640 normal distribution described the shape of the conduction delay distribution well (least-squares

641 fit $\mathrm{R}^{2}=0.9988$ ). We therefore used mean and standard deviation of a log-normal distribution to 642 parameterize the conduction delays for the polychronous network models. 
644 Conduction delays along L4 spiny neuron axons were estimated in the same way. We measured

645 the distribution of pathlength distances of 14 complete reconstructions of the intracortical axonal

646 arbor of L4 neurons labeled in vivo (Narayanan et al., 2015) and multiplied pathlengths by a

647 conduction velocity of $0.3 \mathrm{~mm} / \mathrm{ms}$ to obtain the conduction delay distribution.

648

649 The conduction time along long-range axons from HVC to RA was measured from whole-cell

650 membrane potential recordings of HVC premotor neurons as the difference between the onset

651 time of antidromic stimulation in RA and action potential onset. The action potential onset was

652 defined by calculating the second derivative of the membrane potential between 0-20 ms after

653 stimulation and determining the first upward threshold crossing, where the threshold was set as

654 the minimum of either 3 standard deviations of the second derivative or $400 \mathrm{mV} / \mathrm{ms}^{2}$. To

655 determine the threshold between putative groups of conduction delays, we used k-means

656 clustering with two groups. The pathlength of the long-range axon of HVC premotor neurons

657 was measured from the soma to the first bifurcation of the axon as it entered RA. The average

658 conduction velocity of unmyelinated descending axons was calculated by dividing the average

659 descending pathlength by the average conduction time of the second mode of the conduction

660 time distribution measured as described above. We then used a simple biophysical model relating

661 the diameter of unmyelinated axons to conduction velocity (Hodgkin and Huxley, 1952;

662 Rushton, 1951):

$$
u=c \sqrt{d}
$$

663 Here, $u$ is the conduction velocity, $d$ the axon diameter, and $c$ a constant. We determined $c$ using 664 the average conduction velocity and average diameter of putative unmyelinated descending 
axons and assumed that this constant is the same for unmyelinated local axons of HVC premotor neurons (i.e., that the basic biophysical properties underlying action potential propagation are the same). We then calculated a distribution of conduction velocities given the observed distribution of diameters of local axonal projections. To estimate the distribution of conduction times to synapses onto other HVC premotor neurons, we used a Monte Carlo simulation approach. We stepped through all possible synapse locations along the set of reconstructed axon morphologies of HVC premotor neurons. For each possible location, we calculated the distribution of conduction times to that location given the pathlength to the soma and the estimated distribution of local conduction velocities. We then randomly selected one of the possible conduction times and randomly assigned it as a synapse onto other HVC premotor neurons based on EM measurements of premotor synapse density for each location relative to the soma (Kornfeld et al., 2017). We ran 100 Monte Carlo simulations to obtain a robust estimate of the resulting conduction time distribution to other HVC premotor neurons.

\section{Frequency analysis of burst onset times}

For each syllable in the electrophysiology data set, we determined the syllable length and number of burst onset times occurring during the syllable. In our modeling effort (see Figure $2 \mathrm{~F}$, 3D and 3G), we simulated possible burst onset time distributions by sampling random numbers distributed in time according to the burst density of the model, while preserving the distribution of syllable lengths from the experimental data sets and the number of burst onset times observed during each syllable. For each syllable, we then defined the power spectral density $\mathrm{P}_{\mathrm{s}}$ of the burst times as the absolute magnitude squared of the discrete Fourier transform evaluated at frequencies $f$ between 1 and $300 \mathrm{~Hz}$, in increments of $4 \mathrm{~Hz}$ : 


$$
P_{s}(f)=\left|\sum_{j=1}^{n} \exp \left(2 \pi i f t_{j}\right)\right|^{2}
$$

688 Here, $n$ is the number of bursts in the syllable, and $t_{j}$ the burst onset time of the $j^{\text {th }}$ burst. We then 689 calculated the mean power spectrum across all syllables. In order to obtain a reliable estimate of 690 the predicted power spectrum of each model and its uncertainty, we repeated this procedure 691 10,000 times, and computed the mean and standard deviation at all evaluated frequencies. For 692 comparison with experimental data, we set the confidence interval as \pm 3 standard deviations.

\section{Neuron and synapse models}

695 HVC premotor neurons were modeled as a two-compartment model with a dendritic and somatic 696 compartment (Long et al., 2010). Current injection at the soma triggers sodium channel697 dependent action potentials, while current injection (and synaptic input) to the dendrite 698 compartment triggers an all-or-none calcium spike, which in turn triggers a high-frequency burst of four action potentials at the soma. Ion channels were modeled using the Hodgkin-Huxley

700 formalism. All model parameters are identical to our previous work (Long et al., 2010), except 701 for the following differences: $R_{c}=130 \mathrm{M} \Omega, \mathrm{G}_{\mathrm{s}, \mathrm{L}}=0.05 \mathrm{mS} / \mathrm{cm}^{2}, \tau_{\mathrm{c}}=15 \mathrm{~ms}$. Conductance-based excitatory synapses were modeled according to 'kick-and-decay' dynamics. Upon synaptic

703 release, the synaptic conductance was increased by $\mathrm{G}_{\text {syn }}$, followed by an exponential decay with 704 time constant $\tau_{\mathrm{syn}}=5 \mathrm{~ms}$. The weight $\mathrm{G}_{\mathrm{syn}}$ of individual synapses was drawn from a uniform 705 distribution $\left[0, \mathrm{G}_{\max }\right]$, with $\mathrm{G}_{\max }$ set to $0.05 \mathrm{mS} / \mathrm{cm}^{2}$, a value that leads to a unitary EPSP of $\sim 4$ $\mathrm{mV}$ at the soma (i.e., the average EPSP amplitude is $2 \mathrm{mV}$ (Mooney and Prather, 2005)).

\section{Synfire chain network assembly}


709

710

711

712

713

\section{Feedforward polychronous network assembly}

715 The feedforward polychronous network (Izhikevich, 2006) was assembled in an iterative 716 process. The algorithm was designed to enforce synchrony of the synaptic inputs to the

717 postsynaptic neurons. The timing of presynaptic bursts must be such that the spikes arrive at the 718

The synfire chain network model of HVC premotor neurons was constructed by sequentially connecting 117 nodes with 170 neurons in each node, corresponding to 19,890 neurons in the entire network. Neurons in each node of the synfire chain were connected in a feed-forward allto-all manner to the neurons in the subsequent node. postsynaptic neuron within a narrow time window (discussed below), taking into account the different axonal conduction delays. The number of connections that each neuron can receive was limited to 170 (Kornfeld et al., 2017). The axonal delays of these connections were based on observed delay distributions.

Each iteration consisted of three steps (Figure S1A): (i) simulation of network dynamics to determine the burst onset times of all neurons in the network at the current iteration; (ii) adding feedforward connections constrained by a given conduction delay distribution between 'source neurons' (i.e., presynaptic neurons) and 'target neurons' (i.e., potential postsynaptic targets which do not form outgoing connections in the current iteration); (iii) adding additional neurons into the network. As a result, the feedforward network grows in size and the corresponding population sequence in duration during this iterative process (Figure S1B). In step (i) of each iteration, network dynamics were simulated by activating a set of 200 predefined 'starter neurons' and recording burst onset times of all active neurons in the network. In step (ii), new 
732 feedforward synaptic connections between 'source' and 'target neurons' were added. First, $\mathrm{N}_{\text {new }}$

733 neurons were moved from the set of 'target neurons' in the previous iteration to the set of 'source

734 neurons'. Specifically, these were the 'target neurons' whose simulated burst onset times were

735 within a 2 ms window from the earliest simulated burst onset time of all 'target neurons' (Figure

736 S1C). We then generated a 'synaptic pool' (i.e., a set of conduction delays $\tau_{\mathrm{ax}}$ ) of size $\mathrm{N}_{\text {out }} * \mathrm{~N}_{\text {new }}$

737 (Nout $=170)($ Kornfeld et al., 2017) by sampling from the given distribution of conduction

738 delays. We iterated over all 'target neurons' ordered according to the number of synaptic inputs,

739 starting with the smallest number. For each 'target neuron', we randomly selected a 'source

740 neuron' that fulfilled the polychronization requirement $\left|t_{\text {target }}-\tau_{\text {int }}-\tau_{\text {ax }}-t_{\text {source }}\right| \leq \tau_{\text {sync }}$ using a

741 suitable conduction delay $\tau_{\mathrm{ax}}$ from the 'synaptic pool' (Figure S1D; i.e., requiring that all

742 synaptic inputs to the 'target neuron' arrive within a synchronous time window $2 * \tau_{\text {sync }}$ (here:

743 time window of $1 \mathrm{~ms}$ ). $\mathrm{t}_{\text {source }}$ is the burst onset time of the 'source neuron', $\tau_{\text {int }}$ is the average

744 integration time constant of HVC premotor neurons from onset of the synaptic inputs to burst

745 threshold (set to $5 \mathrm{~ms}$ ) (Long et al., 2010), and $\mathrm{t}_{\text {target }}$ is the burst onset time of the 'target neuron'.

746 If there were multiple $\tau_{\mathrm{ax}}$ allowing a connection between the 'source neuron' and the 'target

747 neuron', the one minimizing the quantity $\left|t_{\text {target }}-\tau_{\text {int }}-\tau_{\mathrm{ax}}-\mathrm{t}_{\text {source }}\right|$ was selected (i.e., only one

748 synapse was placed between a pair of 'source' and 'target neurons'). After placement, this

749 synaptic connection was removed from the 'synaptic pool'. If the number of synaptic inputs to

750 the 'target neuron' reached 170 or no connection from the 'source neurons' could be made given

751 the conduction delays in the 'synaptic pool', it was not considered as a 'target neuron' anymore.

752 In step (iii), neurons were added to the network in order to increase the network from the set of

753 starter neurons to its final size. This step was taken in case there were no more 'target neurons'

754 before the 'synaptic pool' was exhausted. In this case, the set of 'target neurons' was restored to 
755 its state at the beginning of the iteration. A new 'target neuron' (i.e., without any existing

756 incoming or outgoing synaptic connections) was added to the network by placing a synaptic

757 connection with a randomly selected conduction delay $\tau_{\mathrm{ax}}$ from the 'synaptic pool' originating

758 from one of the $\mathrm{N}_{\text {new }}$ 'source neurons' added to the network in this iteration. The putative burst

759 onset time of the new 'target neuron' was defined as: $t_{\text {new }}=t_{\text {source }}+\tau_{\mathrm{ax}}+\tau_{\text {int }}$ (Figure S1E). All

760 other synaptic connections placed in this iteration were removed from the network; the

761 associated conduction delays were moved back into the 'synaptic pool'; and steps (ii) and (iii)

762 were repeated until the 'synaptic pool' was empty. Then, the next iteration was started, and this

763 process repeated until all 20,000 HVC-RA neurons were incorporated into the network. To

764 investigate the effect of conduction delays on sequence generation, we used different conduction

765 delay distributions during network assembly. The delay distribution in the completely assembled

766 network matched the distributions based on observed delays (Figure S1F).

767

768 Simulations

769 During simulations, HVC premotor neurons received additional independent white noise input 770 currents to their somatic and dendritic compartments with zero mean and amplitudes $\mathrm{A}_{\text {soma }}=$

$7710.1 \mathrm{nA}$ and $\mathrm{A}_{\text {dendrite }}=0.2 \mathrm{nA}$, leading to fluctuations of the somatic membrane potential with a 772 standard deviation of $4.2 \mathrm{mV}$ (Long et al., 2010). To account for the white noise currents, the 773 HVC premotor neuron models were treated as a system of stochastic differential equations and 774 solved using the AN3D1 weak $3^{\text {rd }}$ order method (Debrabant, 2010). The simulation time step was 775 set to $0.02 \mathrm{~ms}$. 
777 Each simulation was started by activating the set of 200 'starter neurons' using an excitatory

778 conductance kick with amplitude $300 \mathrm{nS}$ exponential decay with time constant 5 ms (i.e.,

779 simulating synchronous synaptic input). This input was delivered to the 'starter neurons' either

780 synchronously, uniformly distributed over a $7 \mathrm{~ms}$ window, or randomly within a $10 \mathrm{~ms}$ window.

781 In order to minimize transient effects of this activation procedure, the first $50 \mathrm{~ms}$ of simulated

782 activity were discarded. Network activity patterns after this transient period were qualitatively

783 similar between the different activation procedures. To generate burst densities, we ran 50

784 simulations, recorded the burst onset time of each neuron (i.e., the time where the membrane

785 potential at the soma crosses $0 \mathrm{mV}$ for the first time during a burst) and calculated the average

786 number of bursts in $0.75 \mathrm{~ms}$ bins.

787

788

\section{QUANTIFICATION AND STATISTICAL ANALYSIS}

789 All statistical details of experiments can be found in figure legends and the Results section, 790 including the statistical tests used, exact value of $\mathrm{n}$, what $\mathrm{n}$ represents (e.g., number of animals, 791 number of cells, etc.), definition of center, and dispersion and precision measures (e.g., mean, 792 median, SD, SEM, confidence intervals). Significance was defined at a level of 0.05. Normal 793 distribution of data was not assumed. No data were excluded from analysis. Statistical 794 calculations were performed using MATLAB R2016a.

\section{DATA AND SOFTWARE AVAILABILITY}

797 Software and documentation required for setting up and running simulations of the synfire chain

798 and polychronous network models can be downloaded from:

799 https://psu.box.com/s/55gh5tjgpvcxikel4wjfkzxdwyc0s7x4 
bioRxiv preprint doi: https://doi.org/10.1101/864231; this version posted December 5, 2019. The copyright holder for this preprint (which was not certified by peer review) is the author/funder. All rights reserved. No reuse allowed without permission.

800 

SD of the delay distributions in assembled networks match the parameters of the input lognormal distributions. Dashed: identity line.

820 of diameters of descending projection axon and local collaterals of four HVC premotor neurons. systematic errors due to the unknown orientation of the fiber with respect to the imaging plane. 
824 Figure S3. Polychronous network sequences with HVC conduction delays and different

825 initial conditions. Related to Figure 3 and Figure 5. (A) Polychronous network sequence with

826 HVC conduction delays and synchronously active starter neurons. (B) Zoom into the first $50 \mathrm{~ms}$

827 of the sequence in (A). (C) As in (A), starter neuron activity uniformly distributed within a $7 \mathrm{~ms}$

828 window. (D) Zoom into the first $50 \mathrm{~ms}$ of the sequence in (C). (E) As in (A), starter neurons are

829 active at random time points within a $10 \mathrm{~ms}$ window. (F) Zoom into the first $50 \mathrm{~ms}$ of the 830 sequence in (E). (G) Polychronous network sequence shown in Figure 3C (i.e., using HVC

831 pathlength measurements and rodent conduction velocity); starter neurons are active

832 synchronously. (H) Zoom into the first $50 \mathrm{~ms}$ of the sequence in $(\mathrm{G})$. 


\section{REFERENCES}

Abeles, M. (1991). Corticonics: Neural Circuits of the Cerebral Cortex (Cambridge University Press). Amador, A., Perl, Y.S., Mindlin, G.B., and Margoliash, D. (2013). Elemental gesture dynamics are encoded by song premotor cortical neurons. Nature 495, 59-64.

Amari, S. (1972). Learning patterns and pattern sequences by self-organizing nets of threshold elements. IEEE Trans Comp c-21, 1197-1206.

Andalman, A.S., Foerster, J.N., and Fee, M.S. (2011). Control of vocal and respiratory patterns in birdsong: dissection of forebrain and brainstem mechanisms using temperature. PLoS One 6, e25461. Benezra, S.E., Narayanan, R.T., Egger, R., Oberlaender, M., and Long, M.A. (2018). Morphological characterization of HVC projection neurons in the zebra finch (Taeniopygia guttata). J Comp Neurol 526, $1673-1689$.

Bienenstock, E. (1996). On the dimensionality of cortical graphs. J Physiol Paris 90, 251-256.

Bruno, R.M. (2011). Synchrony in sensation. Curr Opin Neurobiol 21, 701-708.

Bruno, R.M., and Sakmann, B. (2006). Cortex is driven by weak but synchronously active thalamocortical synapses. Science 312, 1622-1627.

Budd, J.M., Kovacs, K., Ferecsko, A.S., Buzas, P., Eysel, U.T., and Kisvarday, Z.F. (2010). Neocortical axon arbors trade-off material and conduction delay conservation. PLoS Comput Biol 6, e1000711. Buzsaki, G., and Mizuseki, K. (2014). The log-dynamic brain: how skewed distributions affect network operations. Nat Rev Neurosci 15, 264-278.

Cannon, J., Kopell, N., Gardner, T., and Markowitz, J. (2015). Neural Sequence Generation Using Spatiotemporal Patterns of Inhibition. PLoS Comput Biol 11, e1004581.

Carr, C.E., and Konishi, M. (1988). Axonal delay lines for time measurement in the owl's brainstem. Proc Natl Acad Sci U S A 85, 8311-8315.

Carr, C.E., and Konishi, M. (1990). A circuit for detection of interaural time differences in the brain stem of the barn owl. J Neurosci 10, 3227-3246.

Danish, H.H., Aronov, D., and Fee, M.S. (2017). Rhythmic syllable-related activity in a songbird motor thalamic nucleus necessary for learned vocalizations. PLoS One 12, e0169568.

Debrabant, K. (2010). Runge-Kutta methods for third order weak approximation of SDEs with multidimensional additive noise. Bit 50, 541-558.

Denk, W., Briggman, K.L., and Helmstaedter, M. (2012). Structural neurobiology: missing link to a mechanistic understanding of neural computation. Nat Rev Neurosci 13, 351-358.

Diesmann, M., Gewaltig, M.O., and Aertsen, A. (1999). Stable propagation of synchronous spiking in cortical neural networks. Nature 402, 529-533.

Fee, M.S., Kozhevnikov, A.A., and Hahnloser, R.H. (2004). Neural mechanisms of vocal sequence generation in the songbird. Ann N Y Acad Sci 1016, 153-170.

Fiete, I.R., Senn, W., Wang, C.Z., and Hahnloser, R.H. (2010). Spike-time-dependent plasticity and heterosynaptic competition organize networks to produce long scale-free sequences of neural activity. Neuron 65, 563-576.

Foster, D.J., and Wilson, M.A. (2007). Hippocampal theta sequences. Hippocampus 17, 1093-1099. Galvis, D., Wu, W., Hyson, R.L., Johnson, F., and Bertram, R. (2018). Interhemispheric dominance switching in a neural network model for birdsong. J Neurophysiol 120, 1186-1197.

Gerstner, W., Kempter, R., van Hemmen, J.L., and Wagner, H. (1996). A neuronal learning rule for submillisecond temporal coding. Nature 383, 76-81.

Gibb, L., Gentner, T.Q., and Abarbanel, H.D. (2009). Inhibition and recurrent excitation in a computational model of sparse bursting in song nucleus HVC. J Neurophysiol 102, 1748-1762. Goldman, M.S. (2009). Memory without feedback in a neural network. Neuron 61, 621-634. 
Graber, M.H., Helmchen, F., and Hahnloser, R.H. (2013). Activity in a premotor cortical nucleus of zebra finches is locally organized and exhibits auditory selectivity in neurons but not in glia. PLoS One 8 , e81177.

Hahnloser, R.H., Kozhevnikov, A.A., and Fee, M.S. (2002). An ultra-sparse code underlies the generation of neural sequences in a songbird. Nature 419, 65-70. Hahnloser, R.H., Kozhevnikov, A.A., and Fee, M.S. (2006). Sleep-related neural activity in a premotor and a basal-ganglia pathway of the songbird. J Neurophysiol 96, 794-812. Hamaguchi, K., Tanaka, M., and Mooney, R. (2016). A Distributed Recurrent Network Contributes to Temporally Precise Vocalizations. Neuron 91, 680-693. Helmstaedter, M., Staiger, J.F., Sakmann, B., and Feldmeyer, D. (2008). Efficient recruitment of layer 2/3 interneurons by layer 4 input in single columns of rat somatosensory cortex. J Neurosci 28, 8273-8284. Hirsch, J.A., and Gilbert, C.D. (1991). Synaptic physiology of horizontal connections in the cat's visual cortex. J Neurosci 11, 1800-1809. application to conduction and excitation in nerve. J Physiol 117, 500-544. Hursh, J.B. (1939). Conduction velocity and diameter of nerve fibers. American Journal of Physiology 127, 131-139. Innocenti, G.M., Lehmann, P., and Houzel, J.C. (1994). Computational structure of visual callosal axons. Eur J Neurosci 6, 918-935. Itskov, V., Curto, C., Pastalkova, E., and Buzsaki, G. (2011). Cell assembly sequences arising from spike threshold adaptation keep track of time in the hippocampus. J Neurosci 31, 2828-2834. Izhikevich, E.M. (2006). Polychronization: computation with spikes. Neural Comput 18, 245-282. Jeffress, L.A. (1948). A place theory of sound localization. J Comp Physiol Psychol 41, 35-39. Jin, D.Z., Ramazanoglu, F.M., and Seung, H.S. (2007). Intrinsic bursting enhances the robustness of a neural network model of sequence generation by avian brain area HVC. J Comput Neurosci 23, 283-299. Jun, J.K., and Jin, D.Z. (2007). Development of neural circuitry for precise temporal sequences through spontaneous activity, axon remodeling, and synaptic plasticity. PLoS One 2, e723. Katlowitz, K.A., Picardo, M.A., and Long, M.A. (2018). Stable Sequential Activity Underlying the Maintenance of a Precisely Executed Skilled Behavior. Neuron 98, 1133-1140 e1133. Kleinfeld, D., and Sompolinsky, H. (1988). Associative neural network model for the generation of temporal patterns. Theory and application to central pattern generators. Biophys J 54, 1039-1051. Kornfeld, J., Benezra, S.E., Narayanan, R.T., Svara, F., Egger, R., Oberlaender, M., Denk, W., and Long, M.A. (2017). EM connectomics reveals axonal target variation in a sequence-generating network. Elife 6 . Kosche, G., Vallentin, D., and Long, M.A. (2015). Interplay of inhibition and excitation shapes a premotor neural sequence. J Neurosci 35, 1217-1227.

Laje, R., and Buonomano, D.V. (2013). Robust timing and motor patterns by taming chaos in recurrent neural networks. Nat Neurosci 16, 925-933.

Liewald, D., Miller, R., Logothetis, N., Wagner, H.J., and Schuz, A. (2014). Distribution of axon diameters in cortical white matter: an electron-microscopic study on three human brains and a macaque. Biol Cybern 108, 541-557.

Lombardino, A.J., and Nottebohm, F. (2000). Age at deafening affects the stability of learned song in adult male zebra finches. J Neurosci 20, 5054-5064.

Long, M.A., and Fee, M.S. (2008). Using temperature to analyse temporal dynamics in the songbird motor pathway. Nature 456, 189-194.

Long, M.A., Jin, D.Z., and Fee, M.S. (2010). Support for a synaptic chain model of neuronal sequence generation. Nature 468, 394-399.

Lorteije, J.A., Rusu, S.I., Kushmerick, C., and Borst, J.G. (2009). Reliability and precision of the mouse calyx of Held synapse. J Neurosci 29, 13770-13784. 
Luczak, A., McNaughton, B.L., and Harris, K.D. (2015). Packet-based communication in the cortex. Nat Rev Neurosci 16, 745-755.

Lynch, G.F., Okubo, T.S., Hanuschkin, A., Hahnloser, R.H., and Fee, M.S. (2016). Rhythmic ContinuousTime Coding in the Songbird Analog of Vocal Motor Cortex. Neuron 90, 877-892. Magee, J.C., and Cook, E.P. (2000). Somatic EPSP amplitude is independent of synapse location in hippocampal pyramidal neurons. Nat Neurosci 3, 895-903.

Markowitz, J.E., Liberti, W.A., 3rd, Guitchounts, G., Velho, T., Lois, C., and Gardner, T.J. (2015). Mesoscopic patterns of neural activity support songbird cortical sequences. PLoS Biol 13, e1002158. Mauk, M.D., and Buonomano, D.V. (2004). The neural basis of temporal processing. Annu Rev Neurosci 27, 307-340.

Mello, G.B., Soares, S., and Paton, J.J. (2015). A scalable population code for time in the striatum. Curr Biol 25, 1113-1122.

Miller, R. (1996). Axonal Conduction Time and Human Cerebral Laterality. A Psychobiological Theory. (Amsterdam, The Netherlands: Harwood Academic Publisher).

Miri, A., Daie, K., Arrenberg, A.B., Baier, H., Aksay, E., and Tank, D.W. (2011). Spatial gradients and multidimensional dynamics in a neural integrator circuit. Nat Neurosci 14, 1150-1159.

Mooney, R., and Prather, J.F. (2005). The HVC microcircuit: the synaptic basis for interactions between song motor and vocal plasticity pathways. J Neurosci 25, 1952-1964.

Narayanan, R.T., Egger, R., Johnson, A.S., Mansvelder, H.D., Sakmann, B., de Kock, C.P., and Oberlaender, M. (2015). Beyond Columnar Organization: Cell Type- and Target Layer-Specific Principles of Horizontal Axon Projection Patterns in Rat Vibrissal Cortex. Cereb Cortex 25, 4450-4468. Nottebohm, F., Stokes, T.M., and Leonard, C.M. (1976). Central control of song in the canary, Serinus canarius. J Comp Neurol 165, 457-486.

Okubo, T.S., Mackevicius, E.L., Payne, H.L., Lynch, G.F., and Fee, M.S. (2015). Growth and splitting of neural sequences in songbird vocal development. Nature 528, 352-357.

Pastalkova, E., Itskov, V., Amarasingham, A., and Buzsaki, G. (2008). Internally generated cell assembly sequences in the rat hippocampus. Science 321, 1322-1327.

Peh, W.Y., Roberts, T.F., and Mooney, R. (2015). Imaging auditory representations of song and syllables in populations of sensorimotor neurons essential to vocal communication. J Neurosci 35, 5589-5605. Pehlevan, C., Ali, F., and Olveczky, B.P. (2018). Flexibility in motor timing constrains the topology and dynamics of pattern generator circuits. Nat Commun 9, 977.

Peters, A.J., Chen, S.X., and Komiyama, T. (2014). Emergence of reproducible spatiotemporal activity during motor learning. Nature 510, 263-267.

Picardo, M.A., Merel, J., Katlowitz, K.A., Vallentin, D., Okobi, D.E., Benezra, S.E., Clary, R.C., Pnevmatikakis, E.A., Paninski, L., and Long, M.A. (2016). Population-Level Representation of a Temporal Sequence Underlying Song Production in the Zebra Finch. Neuron 90, 866-876.

Plaza, S.M., Scheffer, L.K., and Chklovskii, D.B. (2014). Toward large-scale connectome reconstructions. Curr Opin Neurobiol 25, 201-210.

Pnevmatikakis, E.A., Soudry, D., Gao, Y., Machado, T.A., Merel, J., Pfau, D., Reardon, T., Mu, Y., Lacefield, C., Yang, W., et al. (2016). Simultaneous Denoising, Deconvolution, and Demixing of Calcium Imaging Data. Neuron 89, 285-299.

Prut, Y., Vaadia, E., Bergman, H., Haalman, I., Slovin, H., and Abeles, M. (1998). Spatiotemporal structure of cortical activity: properties and behavioral relevance. J Neurophysiol 79, 2857-2874.

Rajan, K., Harvey, C.D., and Tank, D.W. (2016). Recurrent Network Models of Sequence Generation and Memory. Neuron 90, 128-142.

Rushton, W.A. (1951). A theory of the effects of fibre size in medullated nerve. J Physiol 115, 101-122. Sabatini, B.L., and Regehr, W.G. (1996). Timing of neurotransmission at fast synapses in the mammalian brain. Nature 384, 170-172. 
Sachidhanandam, S., Sreenivasan, V., Kyriakatos, A., Kremer, Y., and Petersen, C.C. (2013). Membrane potential correlates of sensory perception in mouse barrel cortex. Nat Neurosci 16, 1671-1677. Salami, M., Itami, C., Tsumoto, T., and Kimura, F. (2003). Change of conduction velocity by regional myelination yields constant latency irrespective of distance between thalamus and cortex. Proc Natl Acad Sci U S A 100, 6174-6179.

Schmitt, L.I., Wimmer, R.D., Nakajima, M., Happ, M., Mofakham, S., and Halassa, M.M. (2017). Thalamic amplification of cortical connectivity sustains attentional control. Nature 545, 219-223. Seung, H.S. (2012). Connectome: How the Brain's Wiring Makes Us Who We Are (New York, NY: Houghton Mifflin Harcourt Publishing).

Shu, Y., Duque, A., Yu, Y., Haider, B., and McCormick, D.A. (2007). Properties of action-potential initiation in neocortical pyramidal cells: evidence from whole cell axon recordings. J Neurophysiol 97, 746-760.

Shu, Y., Hasenstaub, A., Duque, A., Yu, Y., and McCormick, D.A. (2006). Modulation of intracortical synaptic potentials by presynaptic somatic membrane potential. Nature 441, 761-765.

Sugihara, I., Lang, E.J., and Llinas, R. (1993). Uniform olivocerebellar conduction time underlies Purkinje cell complex spike synchronicity in the rat cerebellum. J Physiol 470, 243-271.

Swadlow, H.A. (1985). Physiological properties of individual cerebral axons studied in vivo for as long as one year. J Neurophysiol 54, 1346-1362.

Swadlow, H.A. (1994). Efferent neurons and suspected interneurons in motor cortex of the awake rabbit: axonal properties, sensory receptive fields, and subthreshold synaptic inputs. J Neurophysiol 71, 437-453.

Swadlow, H.A., Kocsis, J.D., and Waxman, S.G. (1980). Modulation of impulse conduction along the axonal tree. Annu Rev Biophys Bioeng 9, 143-179.

Swadlow, H.A., Waxman, S.G., and Weyand, T.G. (1981). Effects of variations in temperature on impulse conduction along nonmyelinated axons in the mammalian brain. Exp Neurol 71, 383-389.

Vu, E.T., Mazurek, M.E., and Kuo, Y.C. (1994). Identification of a forebrain motor programming network for the learned song of zebra finches. J Neurosci 14, 6924-6934.

Williams, S.R., and Stuart, G.J. (2002). Dependence of EPSP efficacy on synapse location in neocortical pyramidal neurons. Science 295, 1907-1910.

Yildiz, I.B., and Kiebel, S.J. (2011). A hierarchical neuronal model for generation and online recognition of birdsongs. PLoS Comput Biol 7, e1002303. 

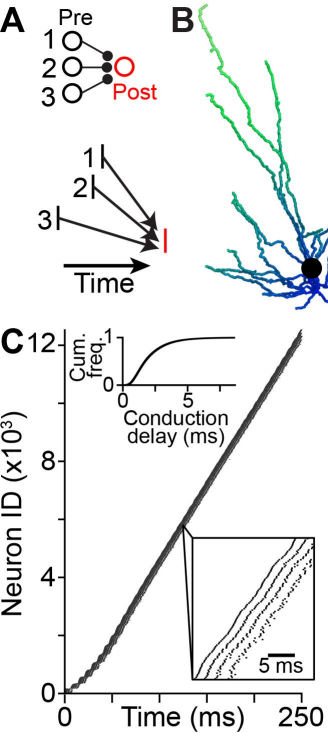

$\frac{D}{2}$

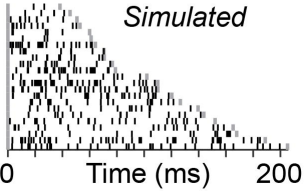

E

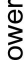

¿

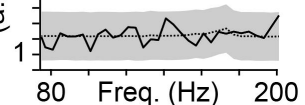

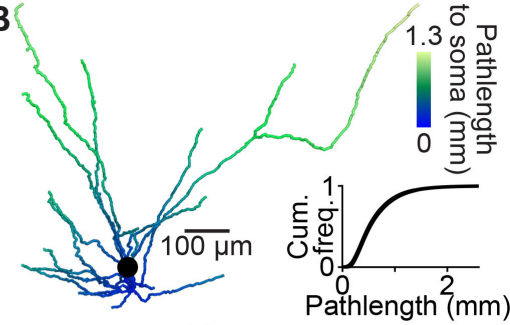

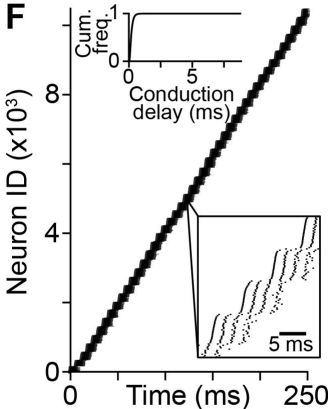

G

ㅇ

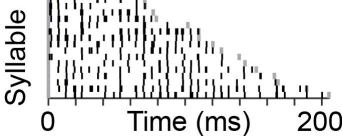

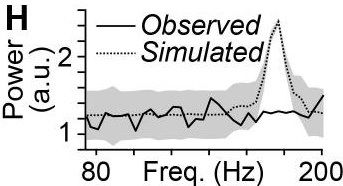




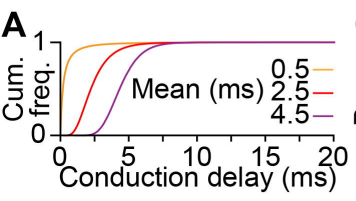

B

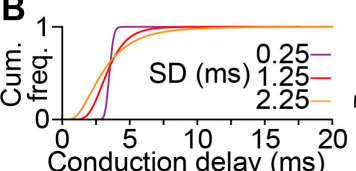

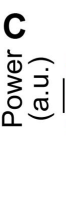

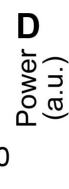

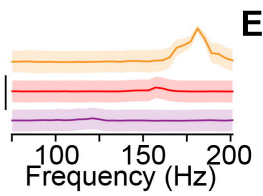

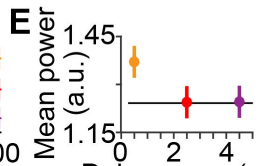

Frequency $(\mathrm{Hz})$

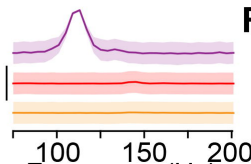

F Frequency $(\mathrm{Hz})$

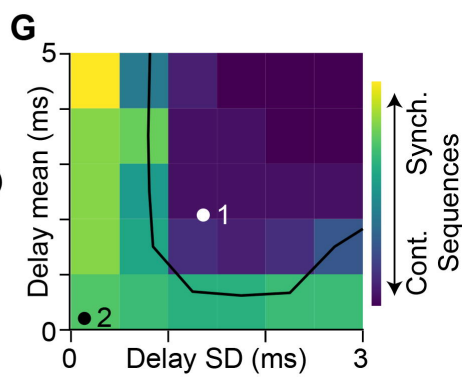


Figure 5 (1.5 columns)

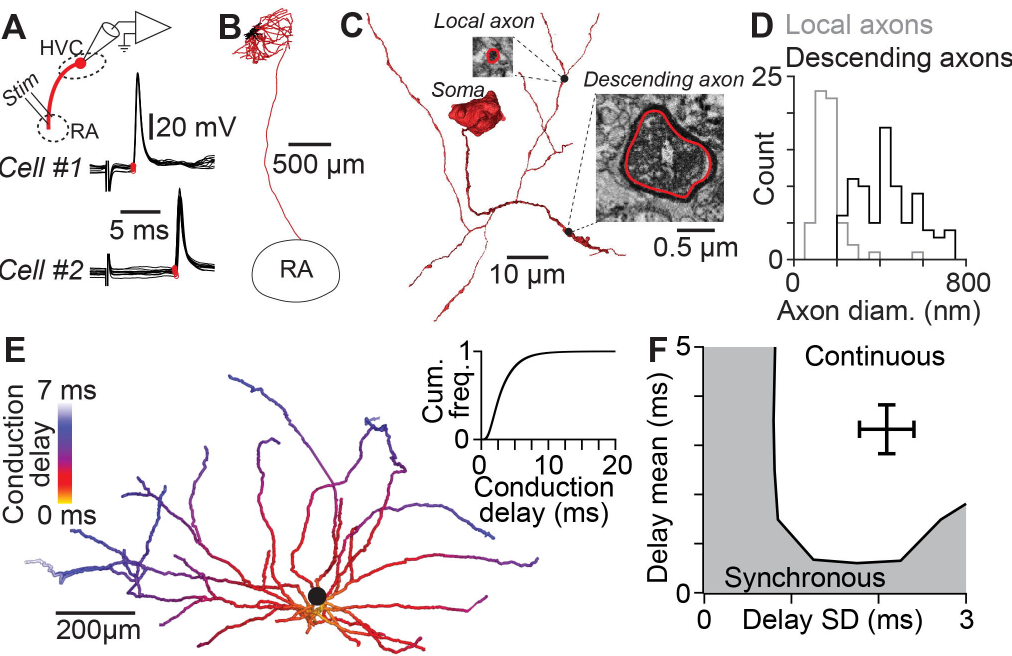


